\title{
Electron kinetics in capacitively coupled plasmas modulated by electron injection
}

Ya Zhang, Yanli Peng, Maria Elena Innocenti, Wei Jiang, Hong-yu Wang, and Giovanni Lapenta

Citation: Journal of Applied Physics 122, 103301 (2017); doi: 10.1063/1.5001892

View online: http://dx.doi.org/10.1063/1.5001892

View Table of Contents: http://aip.scitation.org/toc/jap/122/10

Published by the American Institute of Physics

\section{AlP | Journal of A1Palied Physics}

Save your money for your research.

It's now FREE to publish with us no page, color or publication charges apply.
Publish your research in the

Joumal of Applied Physics

to claim your place in applied

physics history. 


\title{
Electron kinetics in capacitively coupled plasmas modulated by electron injection
}

\author{
Ya Zhang, ${ }^{1}$ Yanli Peng, ${ }_{3}^{2}$ Maria Elena Innocenti, ${ }^{3}$ Wei Jiang, ${ }^{2,3, a)}$ Hong-yu Wang, ${ }^{4, b)}$ \\ and Giovanni Lapenta ${ }^{3}$ \\ ${ }^{1}$ Department of Physics, Wuhan University of Technology, Wuhan 430070, China \\ ${ }^{2}$ School of Physics, Huazhong University of Science and Technology, Wuhan 430074, China \\ ${ }^{3}$ Centre for Mathematical Plasma-Astrophysics, Department of Mathematics, University of Leuven, \\ Leuven 3001, Belgium \\ ${ }^{4}$ School of Physics Science and Technology, Anshan Normal University, Anshan 114007, China
}

(Received 6 April 2017; accepted 9 July 2017; published online 8 September 2017)

\begin{abstract}
The controlling effect of an electron injection on the electron energy distribution function (EEDF) and on the energetic electron flux, in a capacitive radio-frequency argon plasma, is studied using a one-dimensional particle-in-cell/Monte Carlo collisions model. The input power of the electron beam is as small as several tens of Watts with laboratory achievable emission currents and energies. With the electron injection, the electron temperature decreases but with a significant high energy tail. The electron density, electron temperature in the sheath, and electron heating rate increase with the increasing emission energy. This is attributed to the extra heating of the energetic electrons in the EEDF tail. The non-equilibrium EEDF is obtained for strong non-local distributions of the electric field, electron heating rate, excitation, and ionization rate, indicating the discharge has transited from a volume heating ( $\alpha$-mode dominated) into a sheath heating ( $\gamma$-mode dominated) type. In addition, the electron injection not only modifies the self-bias voltage, but also enhances the electron flux that can reach the electrodes. Moreover, the relative population of energetic electrons significantly increases with the electron injection compared to that without the electron injection, relevant for modifying the gas and surface chemistry reactions. Published by AIP Publishing.

[http://dx.doi.org/10.1063/1.5001892]
\end{abstract}

\section{INTRODUCTION}

Low temperature plasmas (LTPs) have long been an important industrial tool given their ability to meet the demands of high throughput and precision. Since, notwithstanding their name, laboratory plasmas are hotter than the environment, energy must be injected to produce and sustain the plasma. Laboratory plasmas can be driven by electric voltage, particle beam injection, or both. One may wonder what plasma can be generated if electron injection (EI) is used alone or together with an electric voltage driving. Such a configuration has been proposed and attracted great attention recently. ${ }^{1-16}$ The groups of Fernsler and Manheimer ${ }^{1,2}$ have studied the fundamental physics of the EI generated plasmas. Chen and $\mathrm{Eden}^{3}$ have realized a microplasma transistor by injecting electrons into the sheath of a plasma with a controllable electron emitter. It is well known that in high aspect ratio contact (HARC) etching, the charging in the trench bottom is a major problem that will lead to notching. ${ }^{4,5}$ Bae and Chang ${ }^{6}$ have introduced an EI emitted from filaments into conventional capacitively coupled plasmas (CCPs). They have shown that the average energy of the electrons to the electrodes can be enhanced significantly, therefore notching effects can be compressed and the surface chemical reactions can be controlled. Lock et al. ${ }^{8}$ have experimentally and theoretically studied the EI generated LTPs, and have reported that the properties of the EI

\footnotetext{
a)Electronic mail: weijiang@hust.edu.cn

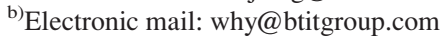

generated LTPs were essentially different from an electrical discharge. Magnetized electron beam generated plasma has also been studied theoretically and experimentally, where plasma uniformity was controlled by the magnetic field modulation. ${ }^{10}$ In addition, the EI generated plasmas in mixed gases have been investigated experimentally ${ }^{7,9}$ and theoretically. ${ }^{11}$ However, in their works, the effect of the gas mixture was mainly discussed. In many cases, mode transitions can be induced by EIs. Chelvam and Raja ${ }^{12}$ have developed a fluid model for a direct-current (DC) microdischarge to study the effect of an external EI from the cathode surface for manipulating the plasma properties. The transient behavior of the microdischarge during the electron injection was examined. It was shown that the maximum average electron energy increased from 18 to over $25 \mathrm{eV}$ in the cathode sheath edge while remaining unchanged in the bulk plasma with the EI. The authors demonstrated that within the scope of the fluid approximation, the electron Joule heating was the main mechanism for the temperature rising to large values in the sheath, since kinetic aspects of the electron motion in the sheath were not accounted for a fluid model. Kushner et al. ${ }^{13}$ used a hybrid plasma equipment model to study the EI controlled ion flux in a radio-frequency (rf) discharge. The authors predicted that the magnitude and energy of the ion flux onto a surface material can be controlled by the modulations of the EI. However, the electron flux as well as the electron energy distribution function (EEDF) have not been discussed. Thus an interesting question arises: how does the $\mathrm{EI}$ affect the electron flux as well as the EEDF? 
Understanding of the electron kinetics, such as the EEDF, is one of the key issues to understand and employ LTPs. The shape of the EEDF is deeply affected by the heating mechanism and characteristics of a discharge. Being able to control and maintain for a long time, a convenient EEDF can enhance the utilization of LTPs significantly. Godyak has written a review of EEDF and average electron energy control in gas discharge plasma. ${ }^{17}$ Haas et al. ${ }^{18}$ used a global model to consider the effect of tailoring of electron energy distributions in a $20 \mathrm{mTorr}$ argon plasma with an rf voltage of $500 \mathrm{~V}$ at $13.56 \mathrm{MHz}$. The principal effect reported was the modification of the rate coefficients for ionization and excitation. Walton's group has experimentally ${ }^{14}$ and numerically ${ }^{15,16}$ studied the EI generated plasmas. Both theory and experiment demonstrated the ability for controlling the EEDF in argon at 25 mTorr immersed in a long cylindrical tube, either by adding an external magnetic field, or by adding small admixtures of nitrogen. The authors used a Langmuir probe to measure the EEDF in their experiment, ${ }^{14}$ while in their numerical work $^{15}$ an electron Boltzmann equation combined with simple fluid equations was employed to model the EEDF. However, the Boltzmann model can only deal with a linear case, where the two-term approximations are adopted. Again their studiess ${ }^{14-16}$ focused on concentrating the EEDF on lower electron energy $(<1 \mathrm{eV})$, obtaining the EI-generated plasmas with a low temperature $(<1 \mathrm{eV})$ and high density $\left(10^{10}-10^{12} \mathrm{~cm}^{-3}\right)$ without any conventionally electrical driving current or voltage source. This kind of low temperature plasmas are largely adopted if plasma processes include plasma processing of polymers and carbon nanostructures. ${ }^{19}$ Having a large population of energetic electrons $(>5 \mathrm{eV})$ in the EEDF could instead be of crucial importance in plasma-gas and plasmasurface chemistry, ${ }^{20}$ because the plasma chemical reactions are controlled by electron energy. This energetic electron flux could provide a new way for transmitting energy to the electrodes and modulating the surface chemistry.

None of the studies mentioned above has been performed using an external EI to modulate the EEDF. However, the EI itself can be an effective mean to modify the EEDF as well. In particular, the first principle kinetic mechanisms behind the formation of an energetic electron flux, as well as their influence on the plasma properties, remain unexplored. In the present work, we investigate the effect of an external EI on the control of the EEDF, plasma electron density and temperature, electron heating rate, and ionization rate, in a capacitive rf argon plasma by using a one-dimensional (1D) particle-in-cell/Monte Carlo collisions (PIC/MCC) model. The calculations are performed at a pressure of $100 \mathrm{mTorr}$, an rf frequency of $13.56 \mathrm{MHz}$, an rf-voltage of $200 \mathrm{~V}$ (amplitude), and a discharge gap of $2 \mathrm{~cm}$. These are typical parameters in conventional low-pressure rf discharges.

\section{MODEL DESCRIPTION}

In this work, a 1D kinetic model is established based on an implicit and electrostatic PIC/MCC method ${ }^{21,22}$ for modulating the EEDF as well as the energetic electron flux by varying the current and energy of an external electron beam. A direct implicit method is used to describe the particle motions. Field equations are derived from direct summation and extrapolation of the equations of particle motion. There are some good reasons that the implicit PIC method is used rather than an explicit one: First, for the most simple explicit electrostatic scheme, the charge and momentum are conserved, but the energy is not conserved. This will lead to the well-known self-heating problem. ${ }^{23,24}$ Thus, the temperature of the electrons will be numerically heated and increased. Whereas, the implicit method allows one to use much larger space and time steps with energy conservation without selfheating. Second, in practice the explicit electrostatic scheme is not able to simulate the high density plasma $\left(\geq 10^{16} \mathrm{~m}^{-3}\right)$. For example, for a 1D PIC/MCC simulation of a CCP with density of $\sim 10^{16} \mathrm{~m}^{-3}$ and a temperature of $\sim 2 \mathrm{eV}$, the explicit scheme will require tens hours on single personal computer. If the density increases to $10^{20} \mathrm{~m}^{-3}$, the computational cost will be 100 times both in space and time, which is not reasonable. Whereas, the implicit scheme can perform the simulation at such high density with reasonably computational cost. ${ }^{25}$ The direct implicit scheme has been introduced by Vahedi to simulate CCP in the early 1990s. ${ }^{26,27}$ The applicability and validity of this direct implicit method have been widely tested not only by our groups, ${ }^{21,28}$ but also by many other researchers in the field of LTPs. ${ }^{29}$

At the boundaries, the electrons and ions are assumed to be absorbed and secondary electron emission (SEE) is not included, since we mainly aim at the effect of an external EI. However, the SEE can play a role in a dielectric flask at fore-vacuum pressures $(0.01-0.1 \mathrm{mTorr}),{ }^{30}$ where the SEE effect becomes stronger with decreasing pressure. In our simulations, the voltage source, rather than the current source, is used to include the electron kinetics. ${ }^{31}$ According to Bae and Chang, ${ }^{6}$ nonzero self-bias voltage can be introduced with EI, so self-bias voltage is self-consistently included by adjusting the electron and ion flux balance to the electrode. In our simulations, self-bias voltage converges to a constant value in several hundreds rf periods. The simulation is conducted with a fixed rf voltage, while the rf power can be modulated by the external EIs. The reason is that the self-bias DC voltage $V_{d c}$ adjusted by the electron and ion flux, and the rf voltage $V_{\text {if }}$ cannot be adjusted simultaneously for a fixed power. The DC self-bias voltage can build on the electrode, which is calculated self-consistently by adjusting the electron and ion flux to the rf powered electrode to be equal. Indeed, a constant rf power has led to unstable results in our simulation. In addition, most researchers ${ }^{27,32-34}$ conducted PIC/MCC simulations with a fixed rf voltage or current and not rf power.

Our simulations are performed for capacitively coupled argon rf discharges at a temperature of $300 \mathrm{~K}$, a pressure of 100 mTorr, with a discharge gap of $2 \mathrm{~cm}$, a rf frequency of $13.56 \mathrm{MHz}$, and a rf voltage of $200 \mathrm{~V}$ (amplitude). An external EI is emitted from the surface of the left electrode with current $J_{e b}$, energy $\epsilon_{e b}$, and a thermal velocity. The injection energy $\epsilon_{e b}$ yields a thermal velocity of $v_{t h}=\sqrt{2 \epsilon_{e b} / m_{e}}$ ( $m_{e}$ is the electron mass). The schematic of the device is shown in Fig. 1, where the EI surface is colored as pink. 


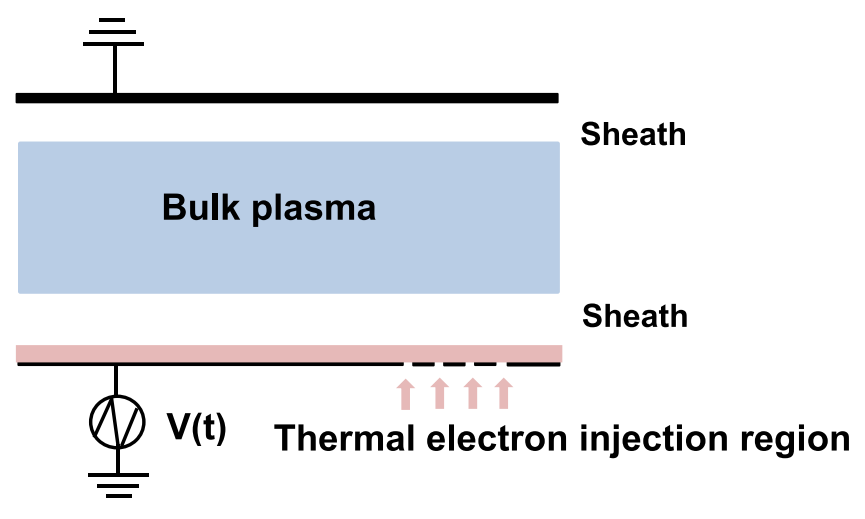

FIG. 1. Schematic illustration of the device: a thermal electron beam injected from the surface of the left electrode.

The initial beam is the monoenergetic electron beam, and the injection beam electron velocity is a thermal velocity which is randomly given with three velocity components. The current $J_{e b}$ and energy $\epsilon_{e b}$ of this external EI are varied in the range of $0.1-5 \mathrm{~A}$ (current density $6.4 \times 10^{3}-3.2 \times 10^{5} \mathrm{~A} \mathrm{~m}^{-2}$ ) and $20-60 \mathrm{eV}$, respectively, which are in the range of realistic values in a CCP experiment. ${ }^{17}$ Notice that "eb" stands for the electron beam. In experiments, the EI can be achieved by the apertures on the electrode.

A standard MCC procedure24 is used to describe the collision processes between different species. Elastic collision processes (the e-Ar, $\mathrm{Ar}^{+}-\mathrm{Ar}$ and charge exchange collisions), excitation and ionization processes from the $\mathrm{Ar}$ ground state and electron-neutral collisions are included into the model. The cross sections are adopted from Ref. 35. We take the initial electron and ion temperatures as $2 \mathrm{eV}$ and $300 \mathrm{~K}$, respectively.

In order to obtain the steady state, the simulations will run for several thousand rf cycles. The simulation time-step is fixed at $10^{-11} \mathrm{~s}$ and 128 cells are adopted for all cases. The numerical stability limits are the Courant condition $\left(d x / d t>V_{t h}\right.$ with the thermal velocity $\left.V_{t h}\right)$ and the empirical condition $\left(d x / d t<10 V_{t h}\right)$. Here $d x$ is the space step and $d t$ is the time step. As the electron and ion numbers increase rapidly due to the EI electrons, a particle merging algorithm is employed when the macro particle number exceeds a certain value (typically 400 per cell). All the simulation results shown below, including the electric field, electron density, electron temperature, electron heating rate and heating power, ionization rate and excitation rate, electron energy distribution function (EEDF), and electron and ion flux will be presented after the simulations have reached a steady state.

\section{RESULTS AND DISCUSSION}

In order to clearly illustrate the effect of an external EI, Fig. 2 compares the space-time varied electric field (a) and (b), electron heating rate (c) and (d), excitation rate (e) and (f), and ionization rate (g) and (h) without EI (left panel) and with EI (right panel) for an EI current of $1 \mathrm{~A}$ (corresponding current density $6.4 \times 10^{4} \mathrm{~A} \mathrm{~m}^{-2}$ ) and an energy of $40 \mathrm{eV}$. The electrode is positioned at $x=0$ and $2 \mathrm{~cm}$. In the absence of the injected EI, the electric field shows wider sheaths, and the nonzero heating rate, excitation rate and ionization rate all occur in the bulk, generating a larger ionization rate in the bulk [see Fig. 2(g)]. The sheaths in Fig. 2(a) are the areas colored in blue and red in the plot. The zero heating rate occurs in sheaths in Fig. 2(c). Thus the discharge is volumetrically heated, excited, and ionized. This is a typical characteristic of a CCP. ${ }^{36}$

With the EI, the electric field takes nonzero values in a narrower region in the sheaths, compared to the case without EI. The sheaths in Fig. 2(b) are the areas colored in blue and red in the plot. Moreover, with considering the EI, the main electron heating, excitation, and ionization process occur in a narrow zone in and around the sheaths. Again the uniformly distributed nonzero values of the heating rate, excitation, and ionization rates [represented by yellow color in Figs. 2(d), 2(f), and 2(h)] in the bulk region, indicate that plasmas are uniformly generated by an external EI with mean free paths of several $\mathrm{cm}$ similar with the discharge gap size. Indeed, in 100 mTorr argon, the electron mean free path for several tens of $\mathrm{eV}$ is of the order of a few $\mathrm{cm}$. The strong non-local distributions of the electric field, electron heating rate, excitation, and ionization rate when including an external EI indicate that the discharge has transited from a volume heating mode ( $\alpha$-mode dominated) into a sheath heating mode ( $\gamma$-mode dominated). ${ }^{17}$ In the $\gamma$-mode, an avalanche of energetic electrons originated from the EI in and around the left sheath causes an intense excitation, ionization, and heating, as clearly seen from Fig. 2 (right panel). This will lead to two-group (cold and thermal) electron populations in the kinetic approach considered here, as clearly seen from the EEDF curves in the presence of the EIs as shown in Fig. 5.

The emission energy of EIs was theoretically (global model) and experimentally proven to have a significant influence on the characteristics of the discharge. ${ }^{18}$ In order to gain a better understanding of the discharge behavior under beam emission, we investigate in Fig. 3 the time-averaged electron density (a), electron temperature (b), electron heating rate (c), and ionization rate (d) at various emission energies for a fixed emission current of $1 \mathrm{~A}$. The results are compared to the case without EI, black line in Fig. 3. The results relative to beam energies of 20,40 , and $60 \mathrm{eV}$ are represented with red-dashed, blue-dotted, and cyan-dash-dotted lines. The electron density increases dramatically with EIs, similar with the experimental and theoretical results in Refs. 12 and 18. The electron temperature in the left sheath increases with EIs, since the injected electrons are significantly heated in the sheath, agreeing with the literature. ${ }^{12}$ The both injected electrons and the EI generated electrons in the sheath will lose their energy through ionization collisions when they reach the bulk in a very short time $(\sim 1 \mathrm{~ns})$, ionization collisions provide an additional cooling mechanism that can reduce the bulk electron temperature, consistent with the literature. ${ }^{18}$ Thus, the electron temperature increases in the sheath while decreases in the bulk with the EIs compared to the case without the EIs. Indeed, without the EIs, two temperature peaks occur near the sheath due to the symmetric non-zero electric field in the sheath [see Fig. 3(a)]. In the 


\section{Left panel:without electron beam}
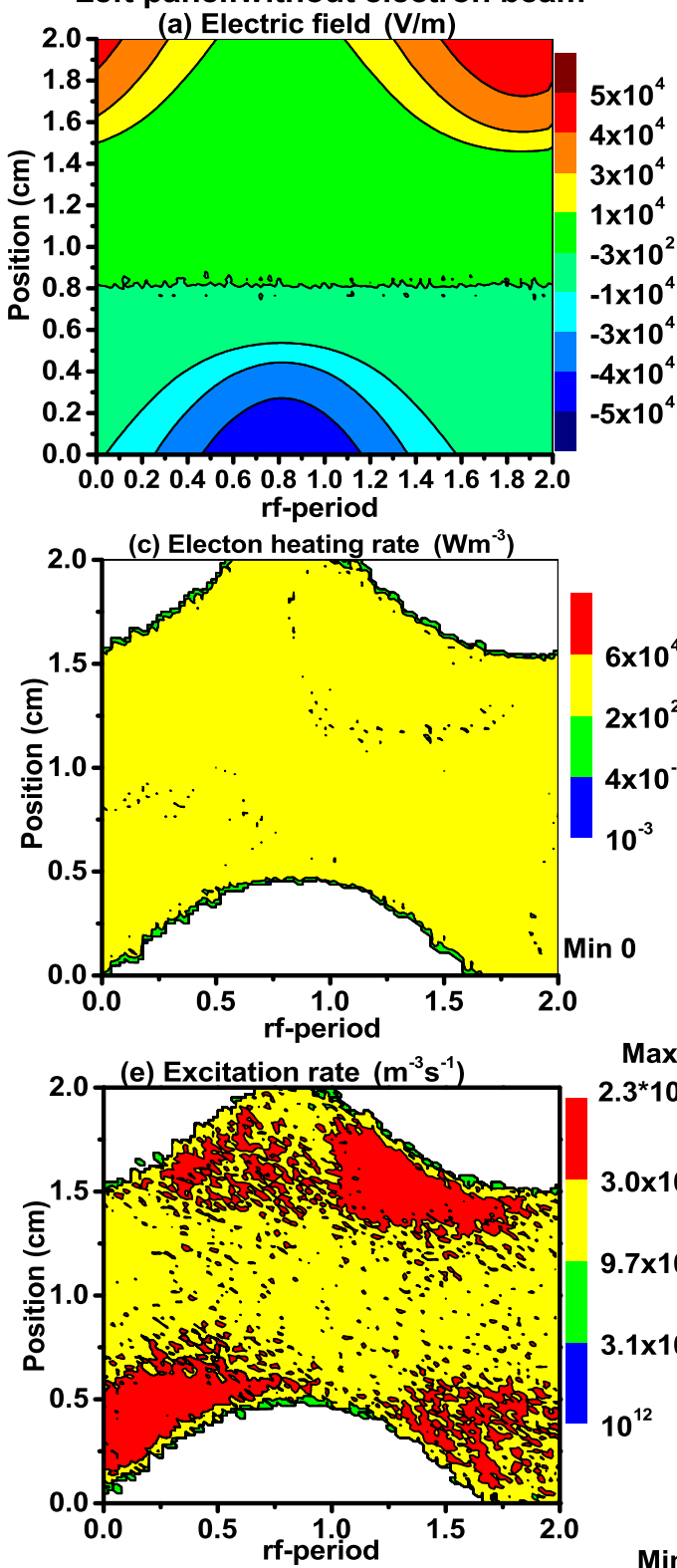

Max $2.3^{\star} 10^{17}$

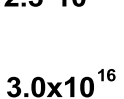

$9.7 \times 10^{14}$
$3.1 \times 10^{13}$ $10^{12}$

Min 0

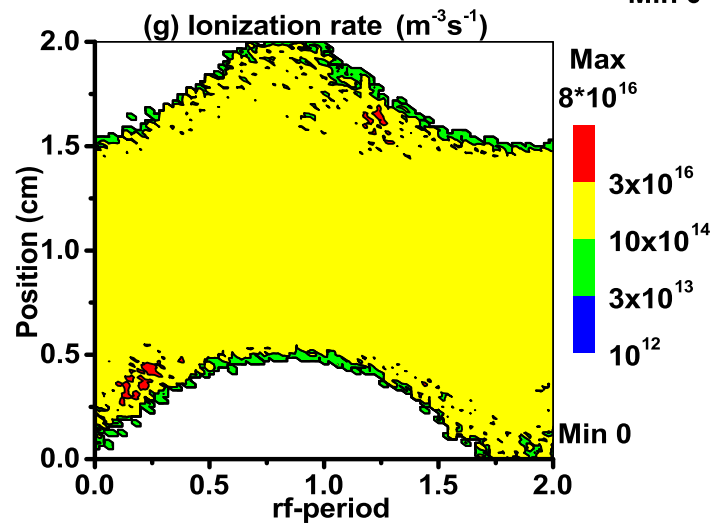

Right panel:with electron beam
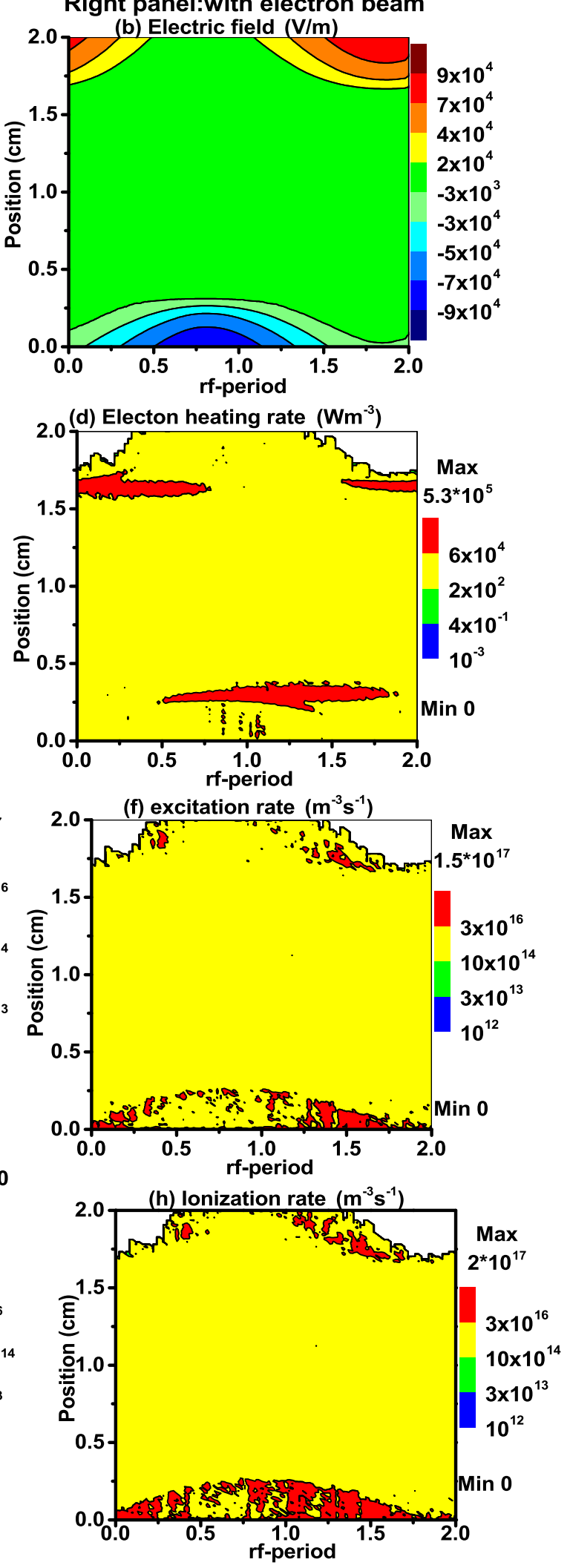

FIG. 2. Left panel: (a), (c), (e), and (g) without the EI; right panel: (b), (d), (f), and (h) with the EI of energy $40 \mathrm{eV}$ and current $1 \mathrm{~A}$. (a) and (b) Electric field, (c) and (d) electron heating rate, (e) and (f) excitation rate, and (g) and (h) ionization rate as functions of time and space.

presence of the EI, the electron heating rate and ionization rate in the sheaths rise rapidly. Again the maximum electron density and heating rate increase with increasing emission energy. The maximum ionization rate in the left sheath occurs at $\epsilon_{e b}=40 \mathrm{eV}$, ascribed to the highest total electron flux (see Fig. 7).

In order to gain a deeper understanding of the discharge behavior as a function of the beam energy, Fig. 4 displays 

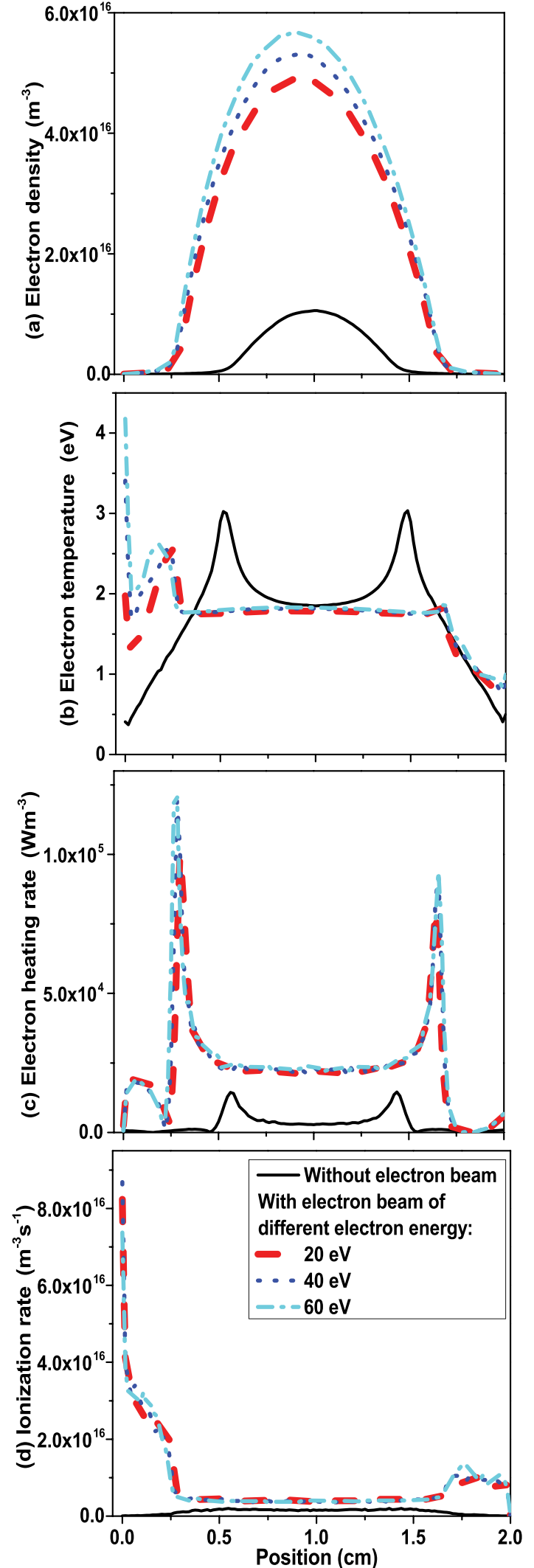

FIG. 3. (a) Electron density, (b) electron temperature, (c) electron heating rate, and (d) ionization rate, without the EI, and with the EIs of three different energies for a fixed current of $1 \mathrm{~A}$.

(a) the self-bias voltage, (b) the space-time averaged electron density and electron temperature in the double $y$ axis, and the heating power (c), for various emission energies of 20 , 40 , and $60 \mathrm{eV}$, with a fixed emission current of $1 \mathrm{~A}$. Note that the values in the absence of the EI are also shown at an emission energy of 0. As shown in Fig. 4(a), the EI introduces a nonzero positive self-bias voltage. With increasing the injection energy, the self-bias voltage becomes larger. The reason is as follows. With the increasing injection energy, energetic electrons produce a higher number of plasma electrons through ionization collisions, hence, the plasma density increases. So a positive self-bias voltage is built at the rf-powered electrode to attract the plasma electrons.

With increasing emission energy from 0 to $20 \mathrm{eV}$, the space-time averaged electron density and temperature, electron heating power, and the total heating power increase relatively sharp, while they increase more slowly as the EI energy rises from 20 to $60 \mathrm{eV}$. The space-time averaged electron temperature, decreases from $\epsilon_{e b}=0$ to $20 \mathrm{eV}$, due to the ionization collisions which induce the dramatically increasing electron density from $\epsilon_{e b}=0$ to $20 \mathrm{eV}$, as seen from Figs. 3(a) and 4(b).

Note that the total heating power consists of the electron and ion heating powers, or beam and rf powers, $P_{\text {total }}=P_{\text {electron }}+P_{\text {ion }}=P_{r f}+P_{e b}$. The heating power of beam $P_{e b}$ is calculated as $P_{e b}=J_{e b} \epsilon_{e b} / e$. Here $e$ is the elementary charge and $\epsilon_{e b}$ has a unit of $\mathrm{eV}$. The heating power is the space integral of the heating rate along the $x$ direction, where the plasma has reached a steady state (thousands of rf-circles). As is clearly seen from Fig. 4(c), the electron, ion and total heating powers increase with increasing the emission energy, and can reach a maximum of 502, 136 , and $638 \mathrm{~W} \mathrm{~m}^{-2}$, respectively. These values are much larger than the pure $\mathrm{rf}$ power of $89 \mathrm{~W} \mathrm{~m}^{-2}$ without the EI, as is clearly seen from the table below. In addition, the table also indicates that the heating power of the injected EI is about ten times smaller than the total heating power. In the absence of the EI, the total heating power is about $89 \mathrm{~W} \mathrm{~m}^{-2}$, however, the total heating power increases by one order of magnitude with the EI. Thus, the total heating power as well as the input rf power are remarkably enhanced with the EI. This is due to the large increase of the electron density, electron heating rate, ionization rate, and electron flux. Indeed, the maximum electron density, ionization rate, heating rate, electron, and ion flux with the EIs, are enhanced by within ten times. Thus the total power is enhanced less than ten times, since the total rf power absorption scales with the density if the energy threshold for electron-ion pair creation will not change much. ${ }^{36}$ Again, the enhance of the rf power has important applications for modulating the EEDF as well as the energetic electron flux. ${ }^{36}$

The space-time averaged EEDFs obtained at different EI energies and fixed EI current of $1 \mathrm{~A}$ are shown in Fig. 5(a). Including the EI, the electron temperature decreases but with a significant high energy tail, indicating a "kinetic effect." The population of middle energy $(>10$ and $<28 \mathrm{eV})$ electrons decreases, while that of the high energy electrons increases $(>28 \mathrm{eV})$. Indeed, without the EI, the "untrapped electrons"37 (thermalized electrons with energy high enough to be able to cross the potential barrier in the sheath) are rapidly lost at the electrode and the EEDF tail disappears, thus the EEDF is a Maxwellian and mainly composed of the "trapped electrons" (electrons bounded in the bulk). 

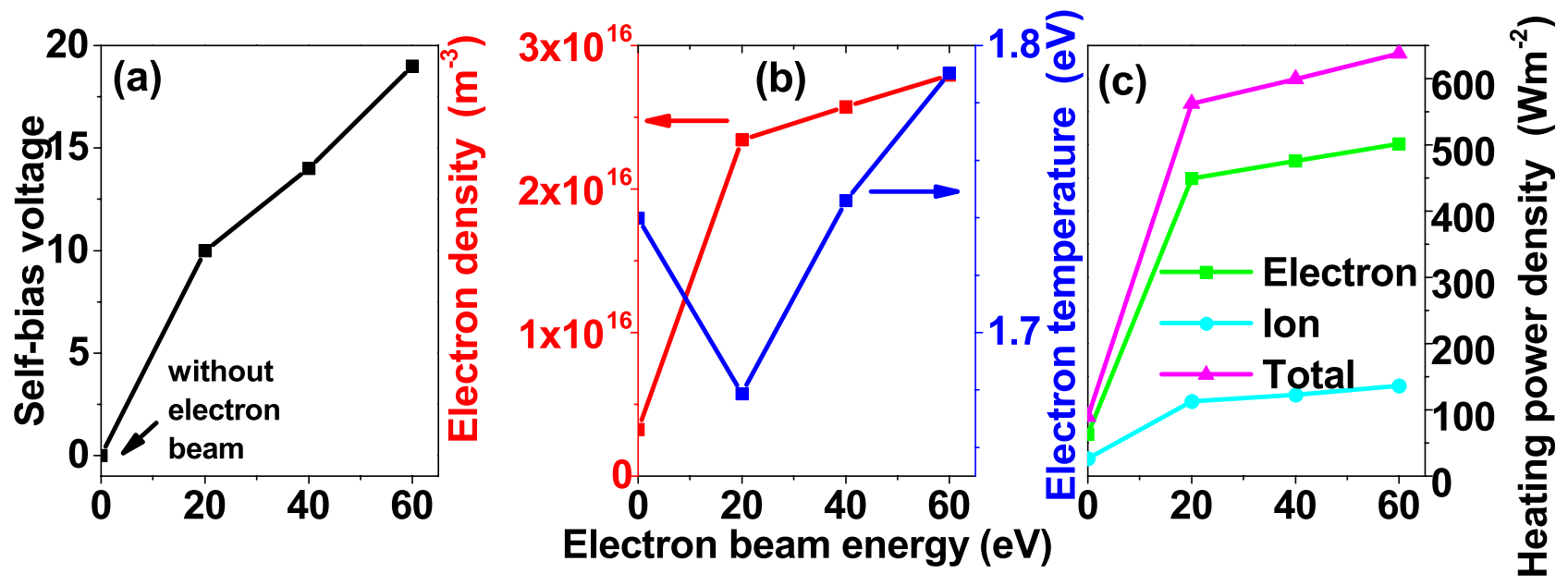

\begin{tabular}{llc}
\hline $\begin{array}{l}\text { Emission } \\
\text { energy }(\mathrm{eV})\end{array}$ & $\begin{array}{l}\text { Beam } \\
\text { power }\left(\mathrm{Wm}^{-2}\right)\end{array}$ & $\begin{array}{l}\text { Total heating } \\
\text { power }\left(\mathrm{Wm}^{-2}\right)\end{array}$ \\
\hline 0 & 0 & 89 \\
20 & 20 & 562 \\
40 & 40 & 599 \\
60 & 60 & 638 \\
\hline
\end{tabular}

FIG. 4. (a) Self-bias voltage and (b) electron density and temperature with double $y$ axis and (c) electron heating power (square), ion heating power (circle), and total heating power (up-triangle), for various EI energies but a fixed EI current of $1 \mathrm{~A}$. The table displays the EI heating power compared to the total heating power at different emission energies with a fixed current of $1 \mathrm{~A}$. Note that the electron density and temperature are space-time averaged. The electron and ion powers are the space integral of the heating rate along the $x$ direction. The power of electron beams is directly calculated from the energy and current of the injected electrons.

Conversely, in the presence of an external EI the "untrapped electrons" are generated from EI electrons and induce a high energy tail of the EEDF. In particular, there occurs a small peak in the EEDF tail around the electron energy at 20, 40, and $60 \mathrm{eV}$, respectively, for $\epsilon_{e b}=20 \mathrm{eV}, \epsilon_{e b}=40 \mathrm{eV}$, and $\epsilon_{e b}=60 \mathrm{eV}$. This means the EEDF indeed can be modulated by the external EIs, which has important applications in experimental and industrial LTPs. ${ }^{17}$

Figure 5(b) presents the space-time averaged EEDFs for different EI currents and a fixed EI energy of 40 A. Again, with including the EI, the average electron energy decreases but with a significant high energy tail. For $J_{e b}=0.1$ and $1 \mathrm{~A}$, the EEDF has a low energy peak at $0.5 \mathrm{eV}$ that is the same as without the EI. However, a high energy tail occurs, which is preferable for plasma processing of surface modification. With the increasing injection current, from $J_{e b}=0.1$ to $J_{e b}=1$ and $5 \mathrm{~A}$, the high energy tails are elevated. This is because, keeping the energy fixed, a higher current corresponds to a higher number of electrons injected. The injected electron density in all cases is very small $\left(\sim 10^{11}-10^{13} \mathrm{~m}^{-3}\right)$ compared to the plasma electron density.

The higher energy tail has important applications not only in gas phase chemistry but also in surface chemistry.
First, an EEDF tail with a controllable high energy will significantly accelerate the reactions by changing the rate coefficients for excitation and ionization in the gas phase. ${ }^{18}$ Second, the energetic electrons in the EEDF tail will be used to modify the surface chemistry reactions. Fine etching uniformity control or effective notching effect suppression in the actual etching process will become more feasible. ${ }^{4,5}$ For example, as Bae and Chang ${ }^{6}$ have pointed out, in HARC etching the notching effect induced by the charging in the deep trench is a major problem, where the EI can be used as an effective way to mitigate this problem.

The kinetic aspects of electrons striking the electrodes are vital in modifying the surface chemistry, since electron kinetics are important for determining surface reactions. Figure 6 presents the electron and ion fluxes to the left electrode as a function of time, with an EI of energy $40 \mathrm{eV}$ and current $1 \mathrm{~A}$. The table (below Fig. 6) shows the time-averaged percentage of three groups of energetic electrons. The electron flux is strongly time-modulated with most electrons striking the electrode when the sheath around the electrode collapses for a positive rf-voltage. The ion flux is not strongly modulated by time variation in an rf-period, forming a plateau in an rfcircle. The reason is that the electrons are much lighter than 

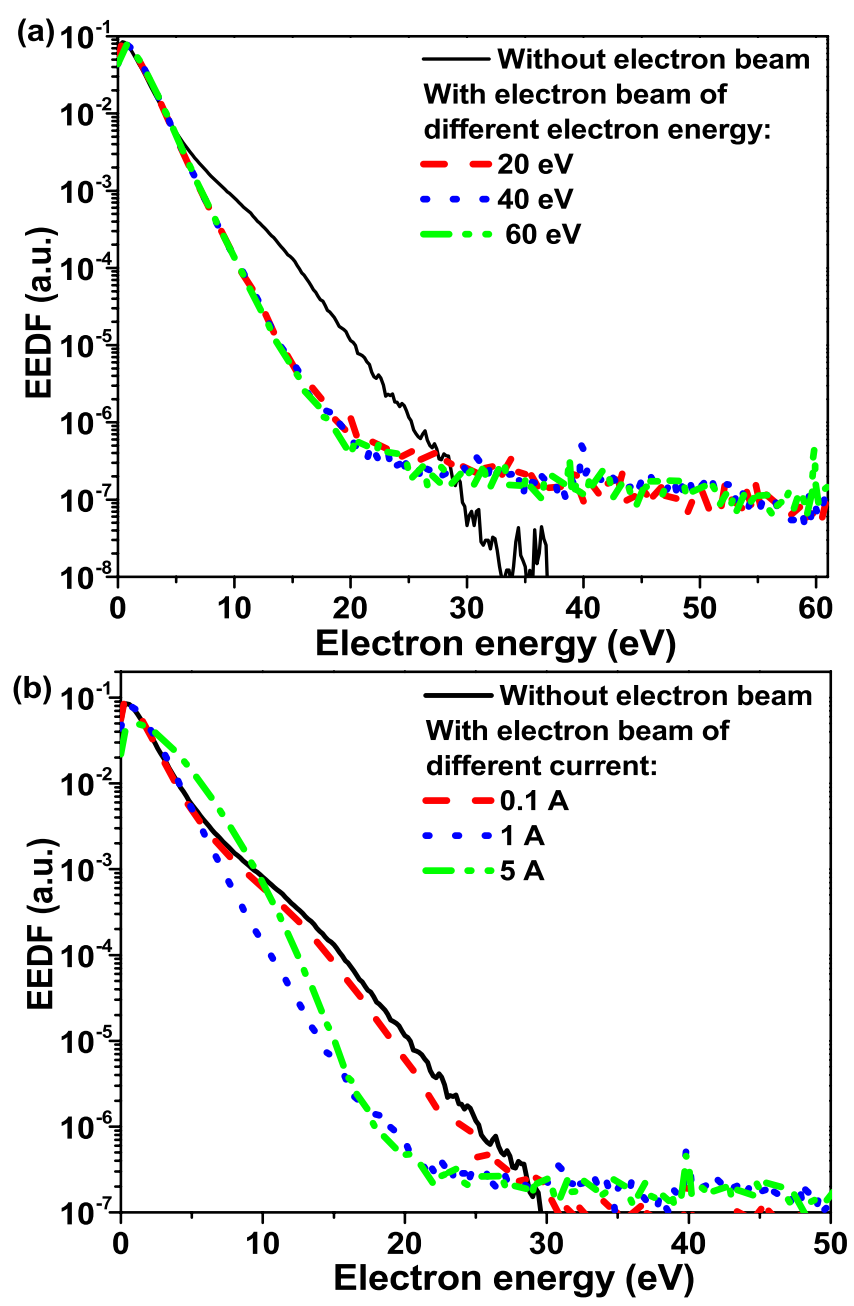

FIG. 5. Space-time-averaged EEDF (a) for various EI energies at a fixed EI current of $1 \mathrm{~A}$ and (b) for various EI currents at a fixed EI energy of $40 \mathrm{eV}$.

ions, and the electrons react to the instantaneous electric field while the ions react to the average electric field. Including the EI, the electron flux to the electrode rises by one order of magnitude, as clearly shown in Fig. 6(b), resulting in much higher time-averaged percentage of energetic electrons as seen from table (b). More significantly, the relative portion of energetic electrons with energy $>5 \mathrm{eV}$ increases by 2 times, with energy $>10 \mathrm{eV}$ increases by 20 times, and $>20 \mathrm{eV}$ increases from $0 \%$ to $53 \%$ with the external EI. Thus, it is possible to optimize the arrival of energetic electrons by adding an external EI into a CCP. These energetic electrons are preferable for the applications of plasma-surface chemistry and low temperature CCP.

Figure 7 displays the electron fluxes to the left electrode as a function of time, for different EI energies [20 eV (solid line), $40 \mathrm{eV}$ (dashed line), and $60 \mathrm{eV}$ (dotted line)] and fixed EI current of $1 \mathrm{~A}$. At a fixed time, the sum of the electron flux is the highest for $\epsilon_{e b}=40 \mathrm{eV}$, higher for $\epsilon_{e b}=20 \mathrm{eV}$, and the lowest for $\epsilon_{e b}=60 \mathrm{eV}$, introducing the maximum ionization rate for $\epsilon_{e b}=40 \mathrm{eV}$, and the lowest ionization rate for $\epsilon_{e b}=60 \mathrm{eV}$ in the sheath [see Fig. 3(d) above]. In addition, the lowest electron flux for $\epsilon_{e b}=60 \mathrm{eV}$ induces the highest electron density [see Fig. 3(a) above] due to the minimum electron loss at the boundary.

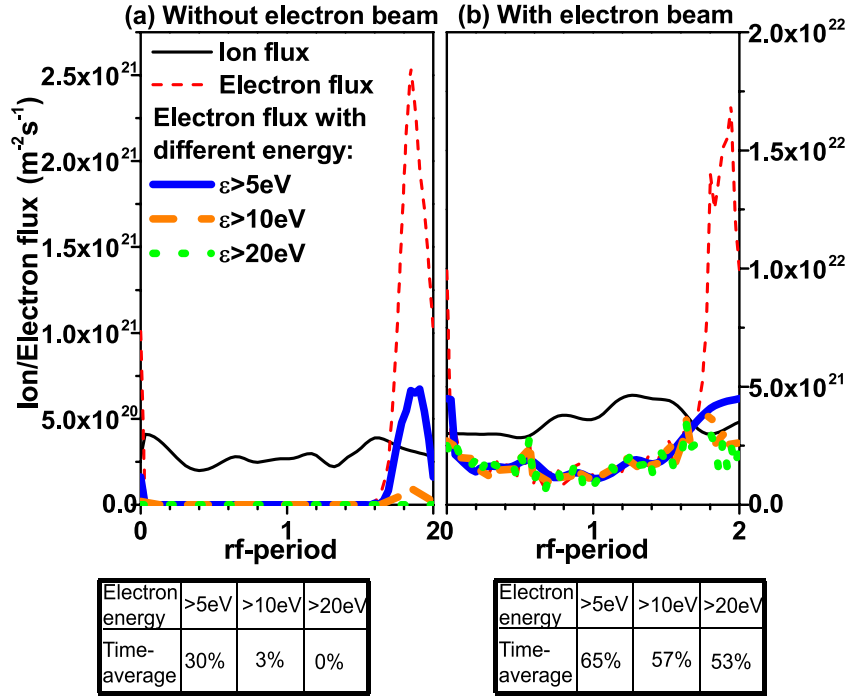

FIG. 6. Electron and ion fluxes to the left electrode as a function of time (a) without the EI and (b) with the EI of energy $40 \mathrm{eV}$ and current $1 \mathrm{~A}$. The table displays the time-averaged percentage of three groups of energetic electrons striking the electrode.

\section{CONCLUSION}

In conclusion, we have used an implicit 1D PIC/MCC model to study the effect of an injected EI on the discharge behaviors of argon $\mathrm{rf} \mathrm{CCP}$ with various emission energies and currents. The pressure is $100 \mathrm{mTorr}$, the discharge gap is $2 \mathrm{~cm}$, the rf frequency is $13.56 \mathrm{MHz}$, and the rf driving voltage is $200 \mathrm{~V}$ in amplitude. Note that the EI needs a small power (several tens of $\mathrm{W}$ ), and the emission current (0.1-5 A) and energy (20-60 eV) taken in consideration can be controlled easily in the laboratory. We have demonstrated the possibility of enhancing the electron heating rate, excitation, and ionization rates in a narrow sheath region by adding the EIs, which in turn controls the EEDF and the energetic electron flux. Again these enhanced ionization collisions lead to much higher electron density but a slightly decreasing electron temperature in bulk while increasing in the sheath. This is because the majority electrons are in the low temperature range (few eV), since the high temperature electrons will lose their energy quickly by inelastic collisions, leading to high electron density and relatively low electron temperature.

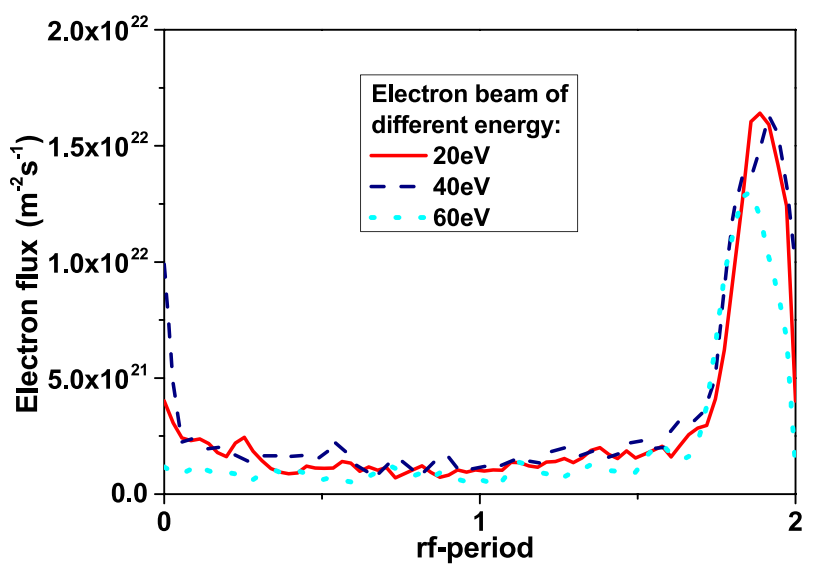

FIG. 7. Electron fluxes to the left electrode as a function of time for different EI energies and a fixed EI current of $1 \mathrm{~A}$. 
At first, we compare the electric field, electron heating rate, excitation, and ionization rates with and without an external EI. With the EI, the heating mode has transited from a volume heating ( $\alpha$-mode dominated) into a sheath heating $(\gamma$-mode dominated).

Second, we investigate the electron density, electron temperature, electron heating rate, and ionization rate for three emission energies of 20,40 , and $60 \mathrm{eV}$, with a fixed emission current of $1 \mathrm{~A}$. The results are compared to the case without EI. The maximum electron density and heating rate increase with the increasing emission energy. The maximum ionization rate in the left sheath occurs at $\epsilon_{e b}=40 \mathrm{eV}$, which is consistent with a highest electron flux to the left sheath.

Third, the self-bias voltage is considered to modulate the plasma properties, where the self-bias voltage is positive with the EI, attracting the enhanced plasma electrons. The space-time averaged electron density, electron temperature, the electron heating power, and the total heating power increase with the increasing emission energy $20-60 \mathrm{eV}$.

Fourth, the controlling effect of the EEDF is examined, which is a long aim of scientists in the field of LTPs, since in the gas phase plasmas the EEDF can determine a variety of plasma-chemical processes. With the EI, the average electron energy decreases but with a significant high energy tail. Without the EI, the EEDF tail disappears, thus the EEDF is a modified Maxwellian. Conversely, the presence of an external EI induces a high energy tail of the EEDF. In particular, there occurs a small peak in the EEDF tail, indicating a significant increase of the "untrapped electrons." The two-group populations of electrons are governed by a strong kinetic effect.

Last but not least, the enhancing effect of the energetic electron number as well as the flux is discussed. The relative portion of energetic electrons with energy $>10 \mathrm{eV}$ increases by 20 times, and $>20 \mathrm{eV}$ increases from $0 \%$ to $53 \%$ when including an external EI. The engineering of an energetic electron flux is of great benefit in plasma-surface and plasma-gas chemistry.

\section{ACKNOWLEDGMENTS}

This work was supported by the National Natural Science Foundation of China (Nos. 11375163, 11405067, and 11275039). M.E.I. is funded by the FWO (Fonds Wetenschappelijk Onderzoek Vlaanderen) postdoctoral fellowship reference $12 \mathrm{O} 5215 \mathrm{~N}$. This work was supported in Belgium by the Exploratory Bilateral co-operation Programme of Tsinghua University-KU Leuven (ISP/15/ 003TS).

${ }^{1}$ R. Fernsler, W. Manheimer, R. Meger, J. Mathew, D. Murphy, R. Pechacek, and J. Gregor, Phys. Plasmas 5, 2137 (1998).
${ }^{2}$ W. M. Manheimer, R. F. Fernsler, M. Lampe, and R. A. Meger, Plasma Sources Sci. Technol. 9, 370 (2000).

${ }^{3}$ H. L. Chen, H. M. Lee, S. H. Chen, Y. Chao, and M. B. Chang, Appl. Catal. B: Environ. 85, 1 (2008).

${ }^{4}$ K. Denpoh and P. Ventzek, J. Vac. Sci. Technol., A 26, 1415 (2008).

${ }^{5}$ W. Jiang, X. Xu, Z.-L. Dai, and Y.-N. Wang, Phys. Plasmas 15, 033502 (2008).

${ }^{6}$ I. Bae and H. Chang, in APS Gaseous Electronics Conference 2016 (2016).

${ }^{7}$ D. Boris, R. Fernsler, and S. Walton, Plasma Sources Sci. Technol. 24, 025032 (2015).

${ }^{8}$ E. Lock, R. Fernsler, S. Slinker, I. Singer, and S. Walton, J. Phys. D: Appl. Phys. 47, 425206 (2014).

${ }^{9}$ E. Lock, T. B. Petrova, G. Petrov, D. Boris, and S. Walton, Phys. Plasmas 23, 043518 (2016).

${ }^{10}$ S. Rauf, A. Balakrishna, A. Agarwal, L. Dorf, K. Collins, D. R. Boris, and S. G. Walton, Plasma Sources Sci. Technol. 26, 065006 (2017).

${ }^{11}$ D. Levko and L. L. Raja, Plasma Sources Sci. Technol. 25, 064003 (2016).

${ }^{12}$ P. K. P. Chelvam and L. L. Raja, J. Appl. Phys. 118, 243301 (2015).

${ }^{13}$ M. J. Kushner, W. Z. Collison, and D. N. Ruzic, J. Vac. Sci. Technol., A 14, 2094 (1996)

${ }^{14}$ D. Boris, G. Petrov, E. Lock, T. B. Petrova, R. Fernsler, and S. Walton, Plasma Sources Sci. Technol. 22, 065004 (2013).

${ }^{15}$ G. Petrov, D. Boris, T. B. Petrova, E. Lock, R. Fernsler, and S. Walton, Plasma Sources Sci. Technol. 22, 065005 (2013).

${ }^{16}$ G. Petrov, D. Boris, E. Lock, T. B. Petrova, R. Fernsler, and S. Walton, J. Phys. D: Appl. Phys. 48, 275202 (2015).

${ }^{17}$ V. Godyak, Phys. Plasmas 20, 101611 (2013).

${ }^{18}$ F. Haas, A. Goodyear, and N. S. J. Braithwaite, Plasma Sources Sci. Technol. 7, 471 (1998).

${ }^{19}$ M. Baraket, S. Walton, E. Lock, J. Robinson, and F. Perkins, Appl. Phys. Lett. 96, 231501 (2010).

${ }^{20}$ F. Iza, J. K. Lee, and M. G. Kong, Phys. Rev. Lett. 99, 075004 (2007).

${ }^{21}$ W. Jiang, H-Y. Wang, Z-H. Bi, and Y-N. Wang, Plasma Sources Sci. Technol. 20, 035013 (2011).

${ }^{22}$ W. Hong-Yu, S. Peng, J. Wei, Z. Jie, and X. Bai-Song, Chin. Phys. B 24, 065207 (2015).

${ }^{23}$ C. K. Birdsall and N. Maron, J. Comput. Phys. 36, 1 (1980).

${ }^{24}$ V. Vahedi and M. Surendra, Comput. Phys. Commun. 87, 179 (1995).

${ }^{25}$ Y. Zhang, W. Jiang, Q. Zhang, and A. Bogaerts, J. Appl. Phys. 115, 193301 (2014).

${ }^{26}$ V. Vahedi, G. DiPeso, C. Birdsall, M. Lieberman, and T. Rognlien, Plasma Sources Sci. Technol. 2, 261 (1993).

${ }^{27}$ V. Vahedi, C. Birdsall, M. Lieberman, G. DiPeso, and T. Ronhlien, Plasma Sources Sci. Technol. 2, 273 (1993).

${ }^{28}$ Y.-X. Liu, Q.-Z. Zhang, W. Jiang, L.-J. Hou, X.-Z. Jiang, W.-Q. Lu, and Y.-N. Wang, Phys. Rev. Lett. 107, 055002 (2011).

${ }^{29}$ J. Carlsson, A. Khrabrov, I. Kaganovich, T. Sommerer, and D. Keating, Plasma Sources Sci. Technol. 26, 014003 (2016).

${ }^{30}$ D. Zolotukhin, V. Burdovitsin, and E. Oks, Plasma Sources Sci. Technol. 25, 015001 (2015).

${ }^{31}$ S. Wilczek, J. Trieschmann, J. Schulze, R. P. Brinkmann, A. Derzsi, P. Hartmann, Z. Donko, and T. Mussenbrock, in APS Gaseous Electronics Conference 2016 (2016).

${ }^{32}$ D. Eremin, R. P. Brinkmann, and T. Mussenbrock, Plasma Processes Polym. 14(4-5) (2017).

${ }^{33}$ A. Derzsi, I. Korolov, E. Schüngel, Z. Donkó, and J. Schulze, Plasma Sources Sci. Technol. 24, 034002 (2015).

${ }^{34}$ J. K. Lee, N. Y. Babaeva, H. C. Kim, O. V. Manuilenko, and J. W. Shon, IEEE Trans. Plasma Sci. 32, 47 (2004).

${ }^{35}$ A. Phelps and Z. L. Petrovic, Plasma Sources Sci. Technol. 8, R21 (1999).

${ }^{36}$ M. A. Lieberman and A. J. Lichtenberg, Principles of Plasma Discharges and Materials Processing (John Wiley \& Sons, 2005).

${ }^{37}$ E. Kawamura, A. Lichtenberg, and M. Lieberman, Plasma Sources Sci. Technol. 17, 045002 (2008). 